\title{
Spatio-temporal features of the Portevin-Le Chatelier effect
}

\author{
G. Ananthakrishna* \\ Materials Research Centre and Centre for Condensed Matter Theory, Indian Institute of Science, Bangalore 560012, India
}

\begin{abstract}
We show that an extension of Ananthakrishna's (AK) model exhibits all the features of the Portevin-Le Chatelier (PLC) effect including the recently observed crossover from a low dimensional chaotic state at low and medium strain rates to a high dimensional power law state of stress drops at high strain rates. The model also displays three types of bands. The dislocation configurations are shown to be in the pinned stated in the chaotic regime (i.e., the region of type $\mathrm{C}$ and $\mathrm{B}$ bands) and at the edge of unpinning in the power law regime where propagating bands are seen.
\end{abstract}

Keywords: The Portevin-Le Chatelier effect; Chaos; Power laws; The PLC bands

\section{Introduction}

Ever since its discovery, explaining the rich spatiotemporal features of the Portevin-Le Chatelier (PLC) effect has remained a challenging problem. The classical explanation of the PLC effect through dynamic strain aging [1] refers to the repeated pinning and unpinning of dislocations due to their interaction with diffusing solute atoms. This translates into a negative strain rate sensitivity (SRS) of the flow stress at macroscopic length scales and is the basic instability mechanism used in most phenomenological models [1]. The collective behaviour of dislocations manifests in the form of bands. On increasing strain rates or decreasing the temperature, one first finds the randomly nucleated type $\mathrm{C}$, then the correlated 'hopping' type B bands and finally the continuously propagating type A bands.

The inherent nonlinearity and the presence of multiple time scales demands a nonlinear dynamical approach which makes the Ananthakrishna's (AK) model [2] a natural candidate for further study. The model reproduces several generic features of the PLC effect, for instance, the negative SRS of the flow stress [2,3]. Moreover, the specific prediction of chaotic stress drops [4] has been subsequently verified using time series analysis [5,6]. Recent studies report an intrigu-

\footnotetext{
* Tel.: +91 80309 2780/2566; fax: +91 803600683

E-mail address: rani@mrc.iisc.ernet.in.
}

ing crossover from a low dimensional chaotic state found at medium strain rates to a power law state of stress drops seen at high strain rates $[7,8]$. A chaotic state involves only a few degrees of freedom, characterized by self-similarity of the attractor and sensitivity to initial conditions while the power law state corresponds to an infinite dimensional state which is a signature of self-organized criticality [9]. As explaining these features requires spatial degrees of freedom not considered in the original AK model, we introduce a spatial coupling term and show that the model reproduces the crossover in the PLC dynamics as also the different types of bands.

\section{The Ananthakrishna's model}

Here, we outline the model in terms of scaled variables in the notation of [10]. The rate equations for the mobile $\rho_{\mathrm{m}}(x, t)$, immobile $\rho_{\mathrm{im}}(x, t)$, and the Cottrell's type dislocation densities $\rho_{\mathrm{c}}(x, t)$ coupled to stress $(\phi)$ rate equation are:

$$
\begin{aligned}
\frac{\partial \rho_{\mathrm{m}}}{\partial t}= & -b_{0} \rho_{\mathrm{m}}^{2}-\rho_{\mathrm{m}} \rho_{\mathrm{im}}+\rho_{\mathrm{im}}-a \rho_{\mathrm{m}}+\phi_{\mathrm{eff}}^{\mathrm{m}} \rho_{\mathrm{m}} \\
& +\frac{D}{\rho_{\mathrm{im}}} \frac{\partial^{2}\left(\phi_{\mathrm{eff}}^{\mathrm{m}}(x) \rho_{\mathrm{m}}\right)}{\partial x^{2}} \\
\frac{\partial \rho_{\mathrm{im}}}{\partial t}= & b_{0}\left(b_{0} \rho_{\mathrm{m}}^{2}-\rho_{\mathrm{m}} \rho_{\mathrm{im}}-\rho_{\mathrm{im}}+a \rho_{\mathrm{c}}\right)
\end{aligned}
$$



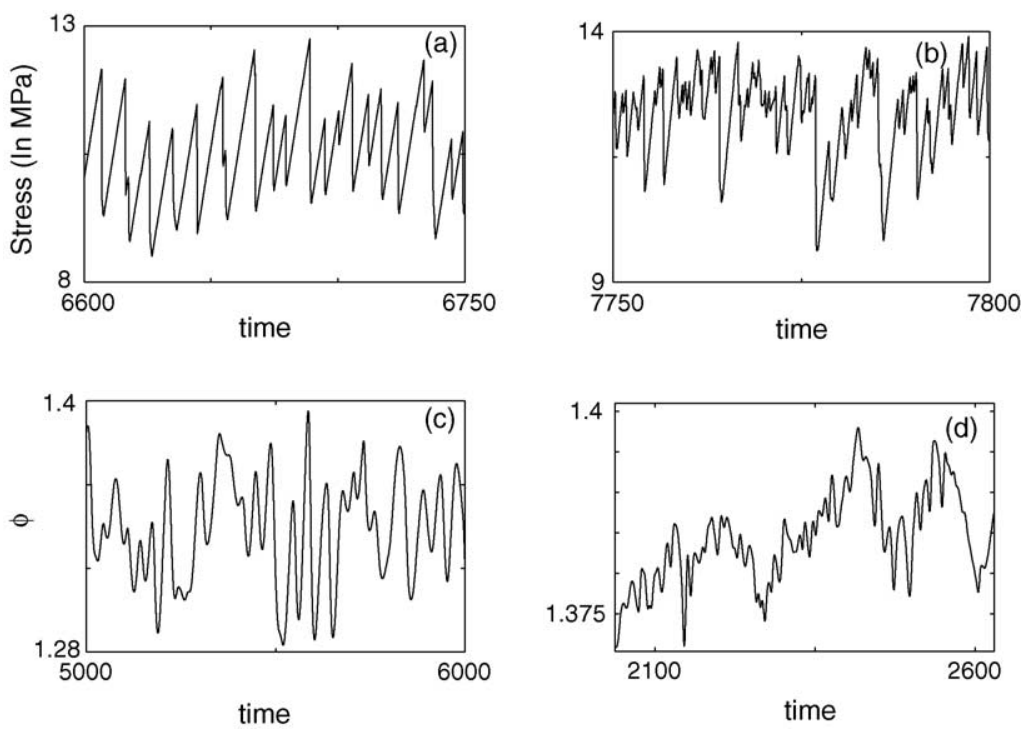

Fig. 1. (a, b) Experimental stress-time series for the chaotic state at (unscaled strain rate) $\dot{\epsilon}_{a}=1.7 \times 10^{-5} \mathrm{~s}^{-1}$ and power law state at $\dot{\epsilon}_{a}=8.3 \times 10^{-5} \mathrm{~s}^{-1}$. (c, d) Stress-time series from the model at $\dot{\epsilon}=120$ and 280, respectively.

$\frac{\partial \rho_{\mathrm{c}}}{\partial t}=c\left(\rho_{\mathrm{m}}-\rho_{\mathrm{c}}\right)$

$\frac{\mathrm{d} \phi(t)}{\mathrm{d} t}=d\left[\dot{\epsilon}-\frac{1}{l} \int_{0}^{l} \rho_{\mathrm{m}}(x, t) \phi_{\mathrm{eff}}^{\mathrm{m}}(x, t) \mathrm{d} x\right]$.

The details of each of the terms have been discussed many times earlier. Here it suffices to say that we have included the formation of locks, annihilation of two mobile dislocations, the remobilization of the immobile dislocation due to stress or thermal activation, the gradual immobilization of mobile dislocations due to solute atoms (defined by $\rho_{\mathrm{c}}$ ), and multiplication of dislocations due to cross-slip. Cross-slip is a natural source of spatial coupling in this model as dislocations generated at a point spread over to the neighboring elements ( $\phi_{\text {eff }}=\phi-h \rho_{\mathrm{im}}^{1 / 2}$ is the effective stress). The various constants have been earlier identified [10].

The PLC state is seen in the domain $10<\dot{\epsilon}<2000$ when other parameters are in the instability limit, here, set to $a=0.8, b_{0}=0.0005, c=0.08, d=0.00006, m=3.0$, $h=0.07$ and $D=0.5$. Eqs. (1)-(4) are discretized into $N$ elements and solved with appropriate boundary conditions.

\section{Dynamics of crossover}

Plots of two experimental stress-strain curves corresponding to the chaotic and power law regimes at medium and high applied strain rates, and time series from the model at intermediate and high values of $\dot{\epsilon}$ are shown in Fig. 1. The similarity of the experimental time series with that of the model at intermediate and high strain rates is clear (note that $\dot{\epsilon}$ is in scaled unit). We summarize the results of dynamical analysis of the model and compare with experiments wherever possible.

(a) We have shown that the experimental time series in Fig. $1 \mathrm{a}$ is chaotic with a finite correlation dimension, $v=2.3$, and a positive Lyapunov exponent. Then, the number of degrees of freedom required for the description of the dynamics is four, consistent with that used in the original model. The geometrical interpretation of these degrees of freedom is that it is the subspace to which the trajectories are confined. The experimental attractor visualized using singular value decomposition $[11,6,10]$ is shown in Fig. $2 \mathrm{a}$ in the space of appropriate combination of the first three principal directions. This is seen to be very similar to the strange attractor obtained from the model in the space of $\rho_{\mathrm{m}}, \rho_{\mathrm{im}}$ and $\rho_{\mathrm{c}}$ (at an arbitrary location,
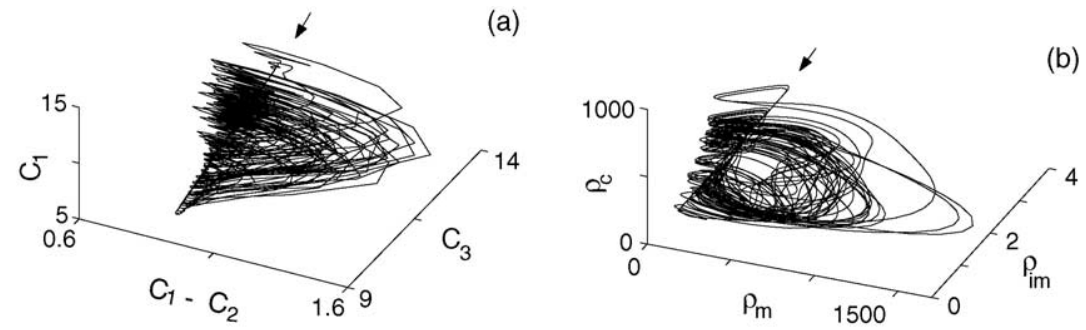

Fig. 2. (a, b) Reconstructed strange attractor for a chaotic experimental stress-time series for $\dot{\epsilon}_{a}=1.7 \times 10^{-5} \mathrm{~s}^{-1}$ and the strange attractor from the model for $\dot{\epsilon}=120$. 


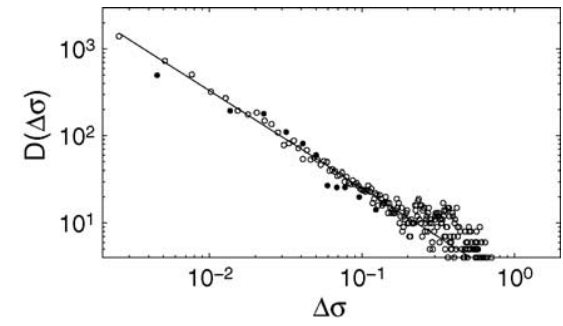

Fig. 3. Distributions of the stress drops from the model( (०), from experiments (•) for $N=1000$ and $\dot{\epsilon}=280$

here $j=50$ and $N=100$ ) shown in Fig. $2 \mathrm{~b}$ for $\dot{\epsilon}=120$ corresponding to the mid chaotic region. Note the similarity about the direction of the arrow which corresponds to the loading direction.

(b) Our analysis shows that the distribution of stress drop magnitudes, $D(\Delta \phi)$, obeys a power law $D(\Delta \phi) \sim \Delta \phi^{-\alpha}$ in the region $260<\dot{\epsilon}<700$. A typical plot for $\dot{\epsilon}=280$ is shown in Fig. 3 (०) along with the experimental points $(\bullet)$ corresponding to Fig. 1a. Both experimental and theoretical points clearly have the same exponent value $\alpha \approx 1.1$.

(c) We have studied the Lyapunov spectrum of the model as a function of $\dot{\epsilon}$. We find the region of chaos to be $35<\dot{\epsilon}<250$ beyond which the largest Lyapunov exponent almost vanishes $\left(\sim 5 \times 10^{-4}\right)$. The region of $\dot{\epsilon}>250$ corresponds to the power law regime of stress drops. While in the chaotic region, the distribution of Lyapunov exponents is quite broad with a small set of of positive exponents, in the power law regime of stress drops, there is a finite density of null exponents (almost vanishing). (d) As null exponents correspond to a marginal state, their finite density in the power law regime implies that most dislocations are perpetually close to the marginal state of unpinning. The configuration of dislocations can be visualized in terms of a parameter which physically corresponds to an effective unpinning stress $\delta=\phi^{\mathrm{m}}$ $\rho_{\mathrm{im}}(j)-a . \delta$ negative, positive and zero correspond, respectively, to the pinned (small values of $\rho_{\mathrm{m}}$ ), unpinned (large values of $\rho_{\mathrm{m}}$ ) and edge of unpinning states. Fig. 4 shows the dislocation configurations just before and after a typical yield drop both in the chaotic and power law state [12]. From Fig. 4, it is clear that while most dislocations are in the pinned state in the chaotic regime, in the power law regime, most dislocations are at the threshold of unpinning, both before and after the yield drop.

(e) For strain rates, $30 \leq \dot{\epsilon}<70$, we get uncorrelated static dislocation bands [13] while for $70 \leq \dot{\epsilon}<180$, we find hopping bands. At high strain rates, we see continuously propagating bands starting from $\dot{\epsilon}=240$ as can be seen from Fig. 5. It is possible to calculate the velocity of the propagating bands in the high strain rate limit. The coupled set of integro-partial differential equations can be to reduced to the standard form of Fischer-Kolmogorov equation for propagative fronts by projecting onto the subspace of the fast variable (see [10]). Using marginal stability analysis, we get the velocity of the bands $v_{\mathrm{b}}=$ $2 \sqrt{\frac{D \dot{\epsilon}}{\bar{\rho}_{\mathrm{m}} \rho_{\mathrm{im}}}\left(\frac{\dot{\epsilon}}{\bar{\rho}_{\mathrm{m}}}-a-\rho_{\mathrm{im}}\right)}$, where $\bar{\rho}_{\mathrm{m}}=\int \rho_{\mathrm{m}}(x) \mathrm{d} x$. At high $\dot{\epsilon}$, we note that $\bar{\rho}_{\mathrm{m}} \sim \bar{\rho}_{\mathrm{m}}^{*}$ is nearly constant as $\bar{\rho}_{\mathrm{m}}^{*}$ is the fixed point value of $\bar{\rho}_{\mathrm{m}}$. The result that the velocity of the bands is proportional to $\dot{\epsilon}$ and $\bar{\rho}_{\mathrm{m}}^{-1}$ appears


Fig. 4. (a, b) Dislocation configurations before and after a typical yield drop in the chaotic regime $\dot{\epsilon}=280$. Note new sites where mobile dislocations are created after the yield drop. (c, d) Dislocation configuration before and after a typical yield drop in the power law regime for $\dot{\epsilon}=280$. 


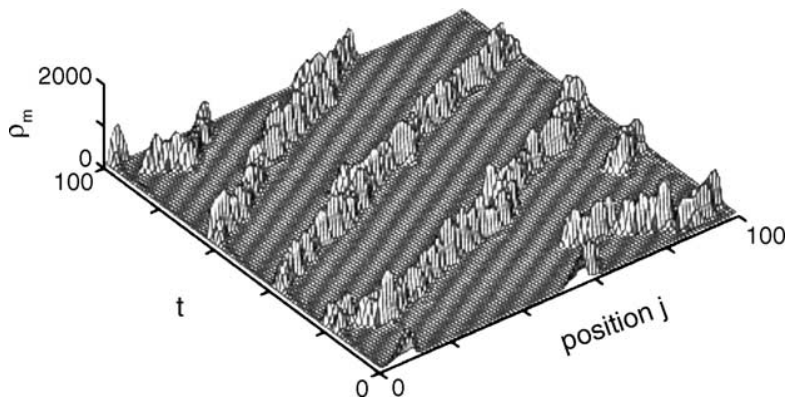

Fig. 5. Propagating type of bands for $\dot{\epsilon}=240$.

to be consistent with the existing experimental results [14]. Also, the fact that hopping type bands belong to the chaotic regime and propagating bands to the power law regime is consistent with the recent studies on $\mathrm{Cu}-\mathrm{Al}$ polycrystals [8]. Curiously, the uncorrelated bands also belong to the chaotic regime.

\section{Discussion and conclusions}

In summary, we have shown that the extended Ananthakrishna's model explains most spatio-temporal features of the PLC effect including the crossover in dynamics as well as the three band types. The dynamics of crossover from chaotic to power law states is fully characterized as resulting from the changes in the nature of Lyapunov spectrum from a small set of positive exponents in the chaotic regime to a dense set of null exponents in the power law state of stress drops. Regarding nature of bands, as the model includes the factor $1 / \rho_{\text {im }}$, this not only accounts for spreading of dislocations into regions of low back stress, it also couples length scales and time scales in a dynamical way due to dependence of $\rho_{\mathrm{im}}$ on $\dot{\epsilon}$, and therefore offers an explanation for the incomplete plastic relaxation at high $\dot{\epsilon}$ as resulting from 'slowing down' of internal relaxation. It is interesting to note that the chaotic state corresponds to the uncorrelated $\mathrm{C}$ and hopping $\mathrm{B}$ types of bands while the power law state corresponds to the propagating type of band. In the latter, as zero exponents correspond to a marginal situation, their finite density physically implies that most dislocations are close to criticality. As far as we know, this is first time a scheme has been developed for visualizing dislocation configurations.

\section{Acknowledgements}

I wish to thank Dr. M.S. Bharathi for the collaborative work. This work is supported by Department of Science and Technology, New Delhi, India.

\section{References}

[1] L.P. Kubin, C. Fressengeas, G. Ananthakrishna, in: F.R.N. Nabarro, M.S. Deusbery (Eds.), Dislocations in Solids, vol. 11, North-Holland, Amsterdam, 2002, p. 101.

[2] G. Ananthakrishna, M.C. Valsakumar, J. Phys. D 15 (1982) L171.

[3] S. Rajesh, G. Ananthakrishna, Phys. Rev. E 61 (2000) 3664; S. Rajesh, G. Ananthakrishna, Physica D 140 (2000) 193.

[4] G. Ananthakrishna, M.C. Valsakumar, Phys. Lett. A95 (1983) 69.

[5] G. Ananthakrishna, et al., Scr. Metall. 32 (1995) 1731.

[6] S.J. Noronha, et al., Int. J. Bifurcation Chaos 7 (1997) 2577.

[7] G. Ananthakrishna, et al., Phys. Rev. E 60 (1999) 5455.

[8] M.S. Bharathi, et al., Phys. Rev. Lett. 87 (2001) 165508.

[9] P. Bak, C. Tang, K. Wiesenfeld, Phys. Rev. Lett. 59 (1987) 381; P. Bak, C. Tang, K. Wiesenfeld, Phys. Rev. A 38 (1988) 364.

[10] G. Ananthakrishna, M.S. Bharathi, Phys. Rev. E 70 (2004) 026111.

[11] H.D.I. Abarbanel, Analysis of Observed Chaotic Data, SpringerVerlag, New York, 1996.

[12] M.S. Bharathi, G. Ananthakrishna, Phys. Rev. E 67 (2003) 065104R.

[13] M.S. Bharathi, S. Rajesh, G. Ananthakrishna, Scr. Mater. 48 (2003) 1355.

[14] P. Hähner, et al., Phys, Rev. B 65 (2002) 134109; A. Korbel, et al., Acta Metall. 24 (1976) 921. 